\title{
Cognitive-behavioural group treatment for a range of functional somatic syndromes: randomised trial ${ }^{\dagger}$
}

\author{
Andreas Schröder, Emma Rehfeld, Eva Ørnbøl, Michael Sharpe, Rasmus W. Licht and Per Fink
}

\section{Background}

Many specialty-specific functional somatic syndrome diagnoses exist to describe people who are experiencing so-called medically unexplained symptoms. Although cognitive-behavioural therapy can be effective in the management of such syndromes, it is rarely available. A cognitive-behavioural therapy suitable for group treatment of people with different functional somatic syndromes could address this problem.

\section{Aims}

To test the efficacy of a cognitive-behavioural therapy (Specialised Treatment for Severe Bodily Distress Syndromes, STreSS) designed for patients with a range of severe functional somatic syndromes.

\section{Method}

A randomised controlled trial (clinicaltrials.gov, NCT00132197) compared STress (nine $3.5 \mathrm{~h}$ sessions over 4 months, $n=54$ ) with enhanced usual care (management by primary care physician or medical specialist, $n=66$ ). The primary outcome was improvement in aggregate score on subscales of the 36-item Short Form Health Survey (physical functioning, bodily pain and vitality) at 16 months.

\section{Results}

Participants receiving STress had a greater improvement on the primary outcome (adjusted mean difference $4.0,95 \% \mathrm{Cl}$ 1.4-6.6, $P=0.002$ ) and on most secondary outcomes.

\section{Conclusions}

In the management of functional somatic syndromes, a cognitive-behavioural group treatment was more effective than enhanced usual care.

\section{Declaration of interest}

None.
Functional somatic syndromes such as fibromyalgia, irritable bowel and chronic fatigue syndrome are a major public health issue. These syndromes are prevalent worldwide and in all medical settings, and when severe pose a major burden on those with the syndrome, on health services and on society as a whole. ${ }^{1-4}$ Although psychiatry has promising treatment strategies at its disposal, few people with these conditions are seen by mental health specialists. ${ }^{5-8}$ Instead, their management is typically shared between primary care and secondary care medical services. It consists largely of repeated investigations by a number of specialist medical services, each of which focuses on diagnosing and managing disease in specific bodily systems, and on the provision of symptomatic treatment when no disease is found. ${ }^{7,9-11}$ Better approaches to treatment are available: cognitive-behavioural therapy (CBT) has been found to be effective in a range of functional somatic syndromes. ${ }^{8,12-16}$ However, the fragmented nature of care imposed by different specialist medical clinics and the unacceptability to patients of referral to mental health services pose obstacles to its efficient delivery and uptake., ${ }^{70,17}$

To address these barriers to treatment we developed a complex intervention based on CBT, entitled Specialised Treatment for Severe Bodily Distress Syndromes (STreSS). This was designed as a common treatment for patients with a variety of severe functional somatic syndromes (grouped under the unifying diagnostic category of bodily distress syndrome, multi-organ type), ${ }^{18,19}$ to be suitable for delivery as a group treatment in a general hospital setting. In this study we aimed to compare the efficacy of STreSS for patients with severe functional somatic syndromes with that of enhanced usual care on self-reported physical health 16 months after randomisation.

'See editorial, pp. 444-445, this issue.

\section{Method}

The study took place at a university general hospital in Aarhus, Denmark. In advance of the project, information about a novel model for the assessment and treatment of functional somatic syndromes was sent to all primary care physicians and hospital wards in the western part of Denmark (Jutland), which covers a population of approximately 2 million persons living in both urban and rural areas. We requested the referral of patients with such syndromes and diagnostic analogues, described in our recruitment letter as 'patients with a long-term illness course due to medically unexplained or functional somatic symptoms who may have received diagnoses like fibromyalgia, chronic fatigue syndrome, whiplash associated disorder, or somatoform disorder'.

Between March 2005 and December 2006 the case notes of all patients referred were screened for eligibility. People who were considered likely to meet inclusion criteria were invited to undergo a clinical assessment to determine eligibility. To be eligible for participation individuals had to have a chronic (i.e. of at least 2 years duration) bodily distress syndrome of the severe multi-organ type, which requires functional somatic symptoms from at least three of four bodily systems, and moderate to severe impairment in daily living. ${ }^{18,19}$ This unifying definition captures most patients with severe functional somatic syndrome diagnoses, ${ }^{19}$ and has a prevalence of $3-4 \%$ in both primary and secondary care. ${ }^{18}$ We included only adults aged 20-45 years, as we regarded the possibility of improvement to be lower in older people and the exclusion of disease to be more difficult. Patients with severe psychiatric morbidity (psychotic and bipolar disorders, alcohol or drug misuse) were also excluded, although we did include people with other psychiatric disorders (e.g. anxiety, depression) and medical disorders (e.g. diabetes, asthma) if these conditions 
did not explain the somatic symptoms. For practical reasons we excluded people involved in litigation, those who were pregnant and those who were not fluent in the Danish language (operationalised as non-Scandinavian origin).

In a block randomisation protocol (six blocks with 20 individuals per block) participants were randomised by means of a computer algorithm that used predefined concealed random numbers and stratified for gender and psychiatric lifetime comorbidity status. In each block 9 participants were randomised to receive STreSS and 11 to receive enhanced usual care. We used the ratio 9:11 because we expected a higher attrition rate in those allocated to enhanced usual care. Patients and their referring doctors were informed of the assignment through standard letters. The study was approved by the Danish Data Protection Agency and the local research ethics committee. All participating patients gave written informed consent before enrolment.

\section{Treatments}

\section{Enhanced usual care}

Figure 1 depicts the treatment elements provided to each group. ${ }^{20}$ All participants underwent a thorough biopsychosocial assessment (' $a$ ' and ' $b$ ' in Fig. 1) prior to randomisation to ensure that their symptoms were not due to an undiagnosed medical condition. The assessment included a review of all clinical records; the semi-structured psychiatric interview Schedule for Clinical Assessment in Neuropsychiatry (SCAN), ${ }^{21}$ which includes an extensive section about physical symptoms; a physical and neurological examination; and a laboratory screening battery. In cases in which the clinical assessment suggested an undiagnosed medical condition we consulted a relevant specialist, or requested further diagnostic tests before the person was considered eligible for participation. All patients were given individualised information about the nature, course and treatment options for their symptoms ('b'). We informed each patient's primary care and hospital doctors of the patient's diagnosis together with a summary of the medical history ('c'). In cases of comorbid anxiety and depression, written individualised advice on treatment was given to the patient's primary care physician. The assessments were made by the same psychiatrists who delivered the STreSS treatment.

Alongside the information on treatment allocation, participants allocated to enhanced usual care were informed that their primary care physician, assisted by the referring specialist where appropriate, would take responsibility for their further treatment. In Denmark $98 \%$ of the population have a primary care doctor who acts as gatekeeper for the specialised healthcare system. The aim of conveying the patients' diagnosis and medical history to the primary care doctor was to achieve a shift from diagnostic procedures to the management of somatic symptoms and comorbid mental illness. Primary care physicians in Denmark are generally well trained in psychiatric care, and many are part of psychiatric supervision groups. ${ }^{22}$ There was no restriction on the psychological or pharmacological interventions that could be given to these patients, or on new referrals to secondary care services. Since it is likely that the clinical assessment ('a', 'b') and the feedback from it (' $c$ ') enhanced the 'usual care' provided to these patients ('d'), we refer to it as 'enhanced usual care'.

\section{Intervention}

The STreSS intervention consisted of the aforementioned clinical assessment, 'usual care' provided by patients' primary care physician, and several specific additional elements (' $e$ ' to ' $i$ ' in Fig. 1). Patients allocated to STreSS received nine modules of

\begin{tabular}{|c|c|c|}
\hline Timeline & $\begin{array}{l}\text { Specialised treatment } \\
\text { for Severe Bodily Distress } \\
\text { Syndromes (STreSS) }\end{array}$ & $\begin{array}{l}\text { Enhanced usual care } \\
\text { (comparison) }\end{array}$ \\
\hline $\begin{array}{l}\text { Clinical assessment } \\
7 \text { (IQR 5-11) weeks } \\
\text { before randomisation) }\end{array}$ & (a) (b) (C) & (a) (b) (C) \\
\hline $\begin{array}{l}\text { Waiting for block } \\
\text { randomisation }\end{array}$ & (d) & (d) \\
\hline $\begin{array}{l}\text { Randomisation } \\
\text { (time 0) }\end{array}$ & \multicolumn{2}{|c|}{$\begin{array}{c}\text { Baseline measurement } \\
\text { (a few days before randomisation) }\end{array}$} \\
\hline $\begin{array}{l}\text { During treatment } \\
\text { period (0-4 months) }\end{array}$ & 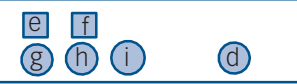 & (d) \\
\hline 4 months & \multicolumn{2}{|c|}{$\begin{array}{l}\text { First outcome measurement } \\
\text { (end of STreSS treatment) }\end{array}$} \\
\hline $\begin{array}{l}\text { During follow-up } \\
\text { period (10-16 months) }\end{array}$ & (d) & (d) \\
\hline 10 months & \multicolumn{2}{|c|}{ Second outcome measurement } \\
\hline $\begin{array}{l}\text { During follow-up } \\
\text { period (10-16 months) }\end{array}$ & (d) & (d) \\
\hline 16 months & \multicolumn{2}{|c|}{ Third outcome measurement (trial end-point) } \\
\hline
\end{tabular}

a Comprehensive lifetime review of case notes and clinical records from primary care physicians, ambulatory care and hospital wards

(b) Comprehensive biopsychosocial assessment, including SCAN diagnostic interview, physical and neurological examination, and laboratory screening battery. At the end of the assessment, patients received individualised information about the nature, course and treatment options for their symptoms

(C) Letter to patient's primary care physician and referring doctor (if not the primary care physician) regarding diagnosis and illness history as well as treatment recommendations in case of comorbid depression or anxiety

(d) 'Usual care' delivered by primary care physician and specialists. No restriction on psychological or pharmacological interventions, or on referral to secondary care or mental health services (for details, see text)

e Letter with management recommendations for functional somatic symptoms sent to primary care physician

Treatment manual, including schedule, symptom diary, educational

$f$ material, worksheets and homework assignment for the nine treatment modules. Patients were handed relevant chapters at the beginning of each module. Non-attending patients received the chapters by post

g) Consultancy service by telephone for primary care physicians and specialists

Nine treatment modules, $3.5 \mathrm{~h}$ each, based on a cognitive-

(h) behavioural approach, delivered in groups of 9 patients by two psychiatrists, at weeks 1, 2, 3, 4, 6, 8, 10, 12 and 16 (see Appendix). Each patient was allowed to receive two supplemental individual

consultations in case of new important physical symptoms or major psychiatric problems. Psychiatrists had at least 2 years of training in cognitive-behavioural treatment, experience with group treatment, and expertise in the field of functional somatic symptoms

(i) Close cooperation with social authorities or the patient's employer, when needed

Fig. 1 Timing and characteristics of treatment elements delivered in each group.

Squares represent fixed elements such as printed materials. Circles represent activities that are flexible, such as clinical assessment. SCAN, Schedule for Clinical Assessment in Neuropsychiatry.

manualised psychotherapy, based on a cognitive-behavioural approach, each of $3.5 \mathrm{~h}$ duration and delivered to groups of nine patients by two psychiatrists. Sessions were given at weeks 1, 2, 3, $4,6,8,10,12$ and 16 after randomisation. Details about the STreSS treatment modules are given in the Appendix. Treatment modules 
of one of the six groups were videotaped and evaluated by two independent psychologists for adherence to the treatment manual. The psychiatrists were consultants (E.R., P.F.) or senior residents (A.S. and one other colleague) in psychiatry with at least 2 years of training in cognitive-behavioural treatment, experience with group treatment and expertise in the field of functional somatic syndromes. The senior residents were supervised by E.R.

The STreSS treatment also included a letter to the patient's primary care doctor (available from the authors on request) which included management recommendations and offered a telephone consultancy service and close cooperation with social authorities and the patients' employer, when needed. The STreSS psychiatrists did not prescribe drug treatment or make referrals to other specialists themselves, but could give advice to the patient's primary care physician to do so. This consultancy service may have further influenced the usual care provided to those allocated to the STreSS intervention, and usual care may therefore have differed between treatment groups.

\section{Usual care and patients' diagnoses}

Details regarding usual care for the period from randomisation to the trial end-point at 16 months were obtained from Danish health registries. We analysed daytime consultations in primary care, contacts with psychiatrists or psychologists in private practice, and use of out-patient and in-patient mental health services. We also recorded use of antidepressants and prescription pain medication from patients' reports at 4 months, 10 months and 16 months. Using the clinical assessment and records we made diagnoses of the most common functional somatic syndromes according to previously reported diagnostic algorithms. $^{19}$

\section{Outcome measures}

Participants completed self-rated measures at baseline (a few days prior to randomisation) and again at 4 months, 10 months and 16 months after randomisation (Fig. 1). Questionnaires were sent by post and administered by independent research assistants who were unaware of the allocation of participants. The primary outcome was the mean change in aggregate score on the 36-item Short Form Health Survey (SF-36) subscales 'physical functioning', 'bodily pain' and 'vitality' from baseline to 16 months, i.e. 1 year after the STreSS intervention was completed. ${ }^{23}$ These three widely used subscales were chosen because they cover key aspects of physical health that are commonly impaired in patients with functional somatic syndromes. ${ }^{24}$ The aggregate score was calculated as the mean of the $z$-scores of the three scales, and transformed into a $t$-score (mean 50, s.d. $=10$, in the 1990 general US population) following the procedure for the calculation of norm-based SF-36 scores. $^{23,25}$ The aggregate score ranges from 15 to 65 and has a mean of 52.5 in the general Danish population. A 4-point increase on the aggregate score equals a change of 0.5 s.d. unit in the study sample and may be regarded as a clinically significant difference. ${ }^{26}$ To facilitate comparison with other trials, we also report the more widely used SF-36 Physical Component Summary (PCS), which in the study protocol was used for power calculation (see below). On this scale, a 4-7 point improvement is regarded as a clinically relevant change. ${ }^{25,27}$

Secondary outcomes were treatment response, defined as an improvement in the SF-36 aggregate score of at least 4 points; improvement in social functioning (SF-36 social functioning scale) and emotional wellbeing (SF-36 mental health scale); and reduction of illness worry (7-item subscale of the Whiteley Index), physical symptoms (90-item Symptom Checklist - Revised somatisation subscale) and severity of depression and anxiety (8-item Symptom Checklist scale). ${ }^{28-30}$

\section{Statistical analysis}

The power calculation was based on the SF-36 PCS scores. ${ }^{25} \mathrm{~A}$ sample size of 120 participants was estimated to provide $83 \%$ power, at the $5 \%$ significance level, to reject the null hypothesis of no difference in improvement between the two groups, when a difference of 5 points on the PCS was assumed and losses to follow-up of $15 \%$ in the STreSS group and 30\% in the comparison group were allowed. However, a validation of the SF-36 questionnaire in the study sample, based on patients' reports at referral, demonstrated serious shortcomings with the SF-36 PCS. ${ }^{31}$ We found an unexpected moderate negative correlation of the physical and mental component summary measures, which are constructed as independent measures. According to the SF-36 manual, a low or zero correlation of the physical and mental components is a prerequisite of their use. ${ }^{23}$ Moreover, three SF-36 scales that contribute considerably to the PCS did not fulfil basic scaling assumptions. ${ }^{31}$ These findings, together with a recent report of problems with the PCS in patients with physical and mental comorbidity, ${ }^{32}$ made us concerned that the PCS would not reliably measure patients' physical health in the study sample. We therefore decided before conducting the analysis not to use the PCS, but to use instead the aggregate score as outlined above as our primary outcome measure. This decision was made on 26 February 2009 and registered as a protocol change at clinicaltrials. gov on 11 March 2009. Only baseline data had been analysed when we made our decision and the follow-up data were still concealed. A post hoc power analysis revealed that power was slightly reduced ( $80 \%$ instead of $83 \%$ ) by the change of primary outcome definition.

All analyses were done on an intention-to-treat basis. We fitted a mixed model with random intercept for the SF-36 aggregate score and all secondary outcomes at baseline, 4 months, 10 months and 16 months, with separate treatment effects calculated for each time point. All analyses were adjusted for gender, age, work status, lifetime psychiatric comorbidity and clinician-rated impairment, and corrected with a cluster effect for treatment group. ${ }^{33}$ Adjustment variables were defined before commencing analyses, and chosen since we regarded them to be potential moderators of change. Using this mixed model, we first tested whether the two groups differed with regard to changes over time on the primary outcome. Next, adjusted change scores from baseline to 4 months, 10 months and 16 months were calculated for each group for the primary and all secondary outcomes. We then calculated comparison effect sizes (adjusted Cohen's $d$ ) for each time point. Finally, we calculated the relative risk for treatment response at 16 months and the number needed to treat (NNT) to achieve one additional treatment response. Further details regarding statistical analysis are available as an online supplement; this supplement also includes a sensitivity analysis in which missing values were replaced by means of multiple imputations. All analyses were done using Stata version 11 for Windows. Since the intervention group was smaller than the comparison group, it was not possible to mask the statistician to group assignment. The trial was registered with clinicaltrials. gov, number NCT00132197.

\section{Results}

Of 278 consecutively referred patients screened for eligibility, 114 were not eligible for the trial and 17 did not agree to participate (Fig. 2). Thus, 147 patients underwent the clinical assessment. Of these, 5 patients declined participation and 22 were not 


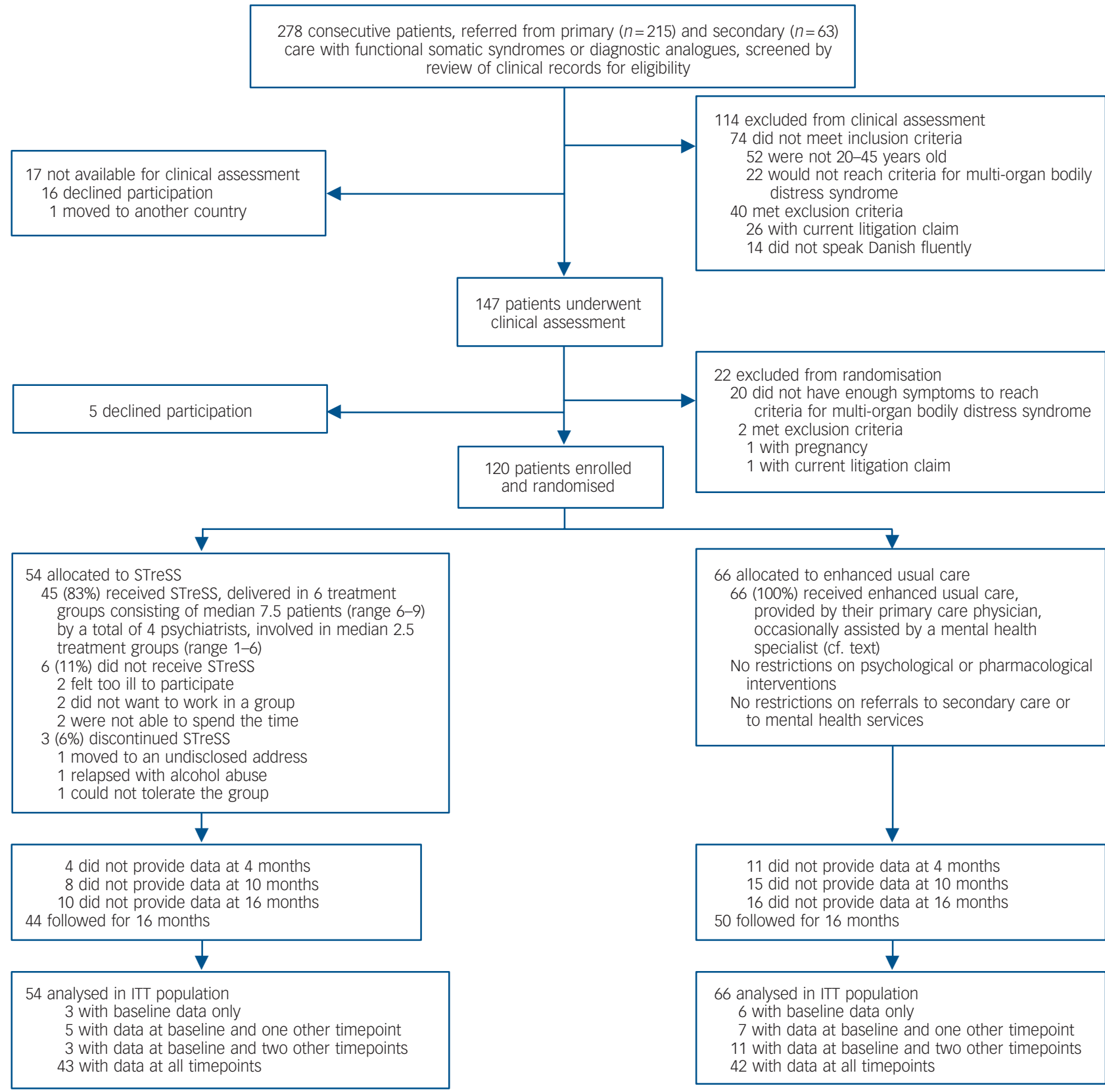

\section{Fig. 2 Trial profile.}

ITT, intention to treat; STreSS, Specialised Treatment for Severe Bodily Distress Syndromes.

eligible. The remaining 120 patients were enrolled and randomly assigned: 66 to enhanced usual care and 54 to STreSS. Outcome data were obtained for 111 patients $(93 \%)$ at one or more time points after baseline and for 94 patients $(78 \%)$ at 16 months (the completers group). Contrary to our expectation, the proportions completing the study were similar in both intervention and usual care groups: $44(81 \%)$ v. $50(76 \%), P=0.45$. An attrition analysis found no significant difference between the completer and non-completer groups with respect to baseline characteristics.

\section{Baseline characteristics of participants and treatment received}

Demographic and clinical characteristics of the study participants are presented in Table 1. Patients' conditions were long-lasting, and a majority were receiving benefits (unemployment, sick leave, disability pension). Most patients qualified for several functional somatic syndrome diagnoses and about a third had a current comorbid mental disorder. All patients fulfilled the diagnostic criteria for at least one somatoform disorder.

Of the 54 patients allocated to STreSS, 3 (6\%) discontinued treatment and $6(11 \%)$ did not attend any of the treatment modules. Specific reasons for leaving the study are given in Fig. 2. Only 3 patients (6\%) explained that they did not want to work in a group, indicating a very high acceptance of the group format. The remaining 45 patients attended on average 8 (interquartile range (IQR) 6-9) treatment modules equalling $28 \mathrm{~h}$ (IQR 27-32) manualised psychotherapy. Psychiatrists spent on average 7.5 treatment hours (sessions) per attending patient. Assessment of recorded sessions indicated that $91 \%$ of treatment was in accordance with the manual. 


\section{Table 1 Patient characteristics}

Intervention Usual care

group $(n=54)$ group $(n=66)$

\begin{tabular}{|c|c|c|}
\hline $\begin{array}{l}\text { Age, years: mean (s.d.) } \\
\text { Gender: female, } n \text { (\%) } \\
\text { Referred from secondary care, } n(\%)\end{array}$ & $\begin{array}{c}35.4(6.3) \\
40(74) \\
8(15)\end{array}$ & $\begin{array}{c}36.2(6.5) \\
55(83) \\
12(18) \\
\end{array}$ \\
\hline \multicolumn{3}{|l|}{ Education, $n$ (\%) } \\
\hline Basic school (years & $31(57)$ & $36(55)$ \\
\hline Further education & $23(43)$ & $30(45)$ \\
\hline \multicolumn{3}{|l|}{ Work status, $n(\%)$} \\
\hline Employed or student & $26(48)$ & $21(32)$ \\
\hline Of these on sick leave & $7(13)$ & $8(12)$ \\
\hline Unemployed & $21(39)$ & $26(39)$ \\
\hline Disability pension or flexible work & $7(13)$ & $19(29)$ \\
\hline \multicolumn{3}{|l|}{ Severe multi-organ bodily distress } \\
\hline syndrome, $n(\%)$ & $54(100)$ & $66(100)$ \\
\hline Number of sub-syndromes: mean (s.d.) & $3.5(0.5)$ & $3.5(0.5)$ \\
\hline \multicolumn{3}{|l|}{ Prevalence, $n(\%)$} \\
\hline $\begin{array}{l}\text { Musculoskeletal tension and pain } \\
\text { syndrome }\end{array}$ & 50 (93) & $62(94)$ \\
\hline Gastrointestinal distress syndrome & $44(81)$ & $53(80)$ \\
\hline Cardiopulmonary distre & $44(81)$ & $52(79)$ \\
\hline General distress syndrome & $53(98)$ & $63(95)$ \\
\hline \multicolumn{3}{|l|}{ Number of functional somatic symptoms: } \\
\hline IIIness duration, years: median (IQR) & $6.7(3-14)$ & $9.5(4-15)$ \\
\hline \multicolumn{3}{|l|}{ Clinician-rated impairment in daily living, $n$ (\%) } \\
\hline Moderate & $17(31)$ & $15(23)$ \\
\hline Severe & 37 (69) & $51(77)$ \\
\hline \multicolumn{3}{|l|}{ Functional somatic syndromes, ${ }^{a, b} n(\%)$} \\
\hline Chronic fatigue syndrome & $30(56)$ & $41(62)$ \\
\hline Fibromyalgia & $38(70)$ & $40(61)$ \\
\hline Irritable bowel syndrome & 19 (35) & $24(36)$ \\
\hline Non-cardiac chest pain & $28(52)$ & $34(52)$ \\
\hline Hyperventilation syndrome & $10(19)$ & $12(18)$ \\
\hline Tension-type headache & 41 (76) & $48(73)$ \\
\hline At least one of the above diagn & $53(98)$ & 60 \\
\hline
\end{tabular}

Current psychiatric comorbidity

(DSM-IV codes), ${ }^{\mathrm{b}} n$ (\%)

Major depressive disorder $(296 . x-296.3 \mathrm{x})^{\mathrm{C}} \quad 9(17) \quad 14(21)$

Dysthymia (300.4) 2 (4) $3(5)$

Anxiety disorder $(300.01,300.21-300.23)^{d} \quad 10(19)$

At least one of the above diagnoses $\quad 16(30) \quad 24(36)$

Lifetime psychiatric comorbidity, $n$ (\%) $\quad 31(57) \quad 40(61)$

Somatoform disorders (DSM-IV codes), ${ }^{\mathrm{b}} n$ (\%)

Somatisation disorder (300.81)

Undifferentiated somatoform disorder

(300.82)

$21(39) \quad 33(50)$

Pain disorder (307.80)

Hypochondriasis (300.7)

At least one of the above diagnoses

$33(61)$

$33(50)$

$22(41) \quad 19(29)$

$6(11) \quad 1(2)$

$54(100) \quad 66(100)$

Health-related quality of life: mean (s.d.)

Physical health aggregate score $(15-65)^{\dagger} \quad 36.4(8.8) \quad 34.6(7.5)$

Social functioning score (0-100) $\quad 57.6(24.6) \quad 54.2(29.7)$

Mental health score (0-100)

$61.6(16.9) \quad 59.3(20.1)$

Illness severity ${ }^{g}$

Physical symptoms score (0-4): mean (s.d. $)^{\text {h }} \quad 1.65(0.67) \quad 1.66(0.71)$

Illness worry score (0-4): median (IQR)

1.21

1.00

$(0.57-1.86) \quad(0.57-1.86)$

0.81

1.25

Depression/anxiety score (0-4): median (IQR)

$(0.50-2.13)$

(0.63-2.00)

IQR, interquartile range.

a. Diagnoses based on functional somatic symptoms in the past 2 years, according

to diagnostic interview and review of clinical records.

b. Allowing more than one diagnosis per patient.

c. Only patients who currently met full criteria for a major depressive episode.

d. Without specific phobias (300.29).

e. Short Form Health Survey (SF-36) questionnaire at baseline; higher scores indicate

e. Short Form
better health.

f. Aggregate score of SF-36 subscales physical functioning, bodily pain and vitality.

$\mathrm{g}$. Baseline measurements; higher scores indicate more severe illness.

h. Scored on the 90-item Symptom Checklist - Revised Somatisation subscale.

Measured using the 7-item Whiteley scale.

j. Measured using the 8-item Symptom Checklist.
We found no difference in the 'usual care' (element ' $d$ ' in Fig. 1) delivered during the 16 months after randomisation between the two groups. Patients had median 7 (IQR 3-13) $v$. median 9 (IQR 5-15) daytime consultations in primary care in the intervention and usual care groups respectively. A similar proportion of participants in the two study groups had contact with a mental health professional: STreSS group $n=11(20 \%)$, median number of consultations 7 (IQR 3-10); usual care group $n=22(33 \%)$, median number of consultations 7 (IQR 3-11). Three participants in the enhanced usual care group and one in the STreSS group received in-patient psychiatric care. At 4 months a slightly higher proportion of patients in the enhanced usual care group $(40 \%, n=22)$ than in the STreSS group $(22 \%, n=11)$ reported daily use of prescription pain medication (two-sample test of proportion $z=1.98, P=0.047)$, whereas there was no difference at 10 months and 16 months $(40-44 \%$ in the usual care group v. 27\% in the STreSS group). We found no difference in the proportion of patients taking antidepressant medication at any time point $(32-36 \%$ in the usual care group $v .35-40 \%$ in the STreSS group).

\section{Main results}

Raw means and standard deviations for the primary outcome and the more widely used SF-36 PCS at baseline and at 4 months, 10 months and 16 months after randomisation are given in Table 2; Fig. 3 shows mean scores with $95 \%$ confidence intervals derived from the regression model for the primary outcome (a) and the secondary outcomes (b) to (f). The treatment groups differed significantly on the primary outcome with regard to changes over time (Wald $\chi^{2}(3)=18.0, P=0.0004$ ), with an adjusted difference in mean SF-36 aggregate score change from baseline to 16 months of 4.0 points $(95 \%$ CI $1.4-6.6, P=0.002)$. Participants in the STreSS group showed a mean improvement in their physical health during that time of 4.0 points (95\% CI 2.0-6.0, $P<0.001)$ or 0.5 s.d. unit, which was the predefined boundary for a clinically relevant change, but we observed no change in the enhanced usual care group ( -0.02 point, $95 \%$ CI -1.6 to 1.6, $P=0.98$ ). Adjusted comparison effect sizes (Cohen's $d$ ) for all time points are also given in Fig. 3. At 16 months a moderate effect size was found for the primary outcome measure $(0.50,95 \%$ CI 0.18-0.83).

The outcome measures by the more widely used SF-36 PCS were similar; these are provided here for comparison and were not part of the primary analysis. The adjusted difference in mean change from

Table 2 Comparison of 36-item Short Form Health Survey aggregate scores (primary outcome) and the Physical

Component Summary (provided to facilitate comparison with other trials) at baseline and follow-up

\begin{tabular}{|c|c|c|c|}
\hline & $\begin{array}{l}\text { STresS group } \\
\text { Mean (s.d.) }\end{array}$ & $\begin{array}{l}\text { Usual care group } \\
\text { Mean (s.d.) }\end{array}$ & $\begin{array}{l}\text { Unadjusted } \\
\text { difference }\end{array}$ \\
\hline \multicolumn{4}{|c|}{ SF-36 aggregate score (physical functioning, bodily pain, vitality) } \\
\hline Baseline & $36.4(8.8)$ & $34.6(7.5)$ & 1.8 \\
\hline 4 months & $39.9(9.6)$ & $33.7(6.6)$ & 6.2 \\
\hline 10 months & $40.7(9.3)$ & $33.4(8.3)$ & 7.4 \\
\hline 16 months & $40.8(10.9)$ & $33.8(8.1)$ & 7.0 \\
\hline \multicolumn{4}{|c|}{ SF-36 Physical Component Summary } \\
\hline Baseline & $32.5(11.0)$ & $31.7(9.6)$ & 0.7 \\
\hline 4 months & $36.7(12.1)$ & $30.2(9.3)$ & 6.4 \\
\hline 10 months & $36.3(12.3)$ & $29.7(10.0)$ & 6.6 \\
\hline 16 months & $38.7(13.0)$ & $29.8(11.0)$ & 8.8 \\
\hline \multicolumn{4}{|c|}{$\begin{array}{l}\text { SF-36, 36-item Short Form Health Survey; STreSS, Specialised Treatment for Severe } \\
\text { Bodily Distress Syndromes. } \\
\text { a. Means are raw values. }\end{array}$} \\
\hline
\end{tabular}



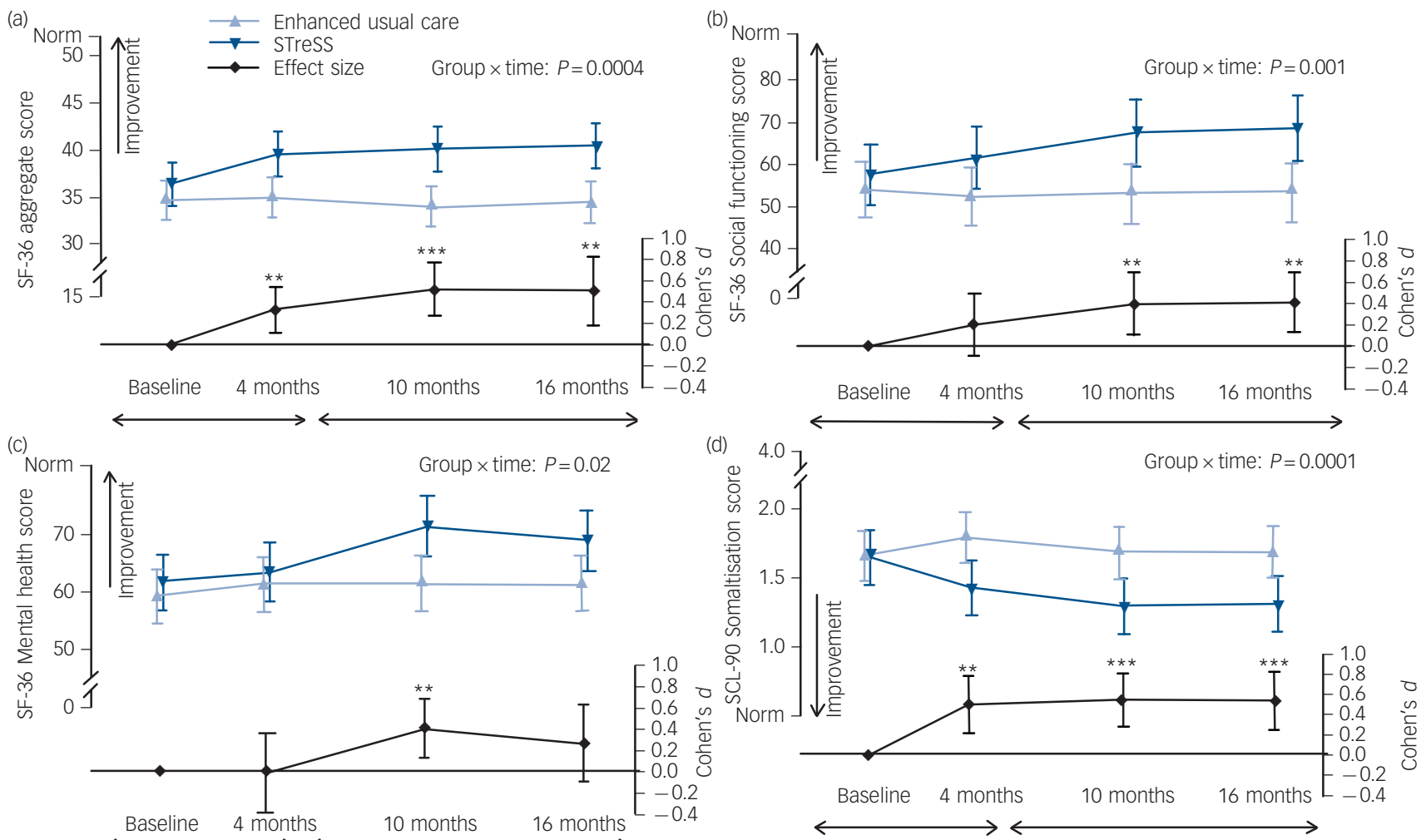

(e)
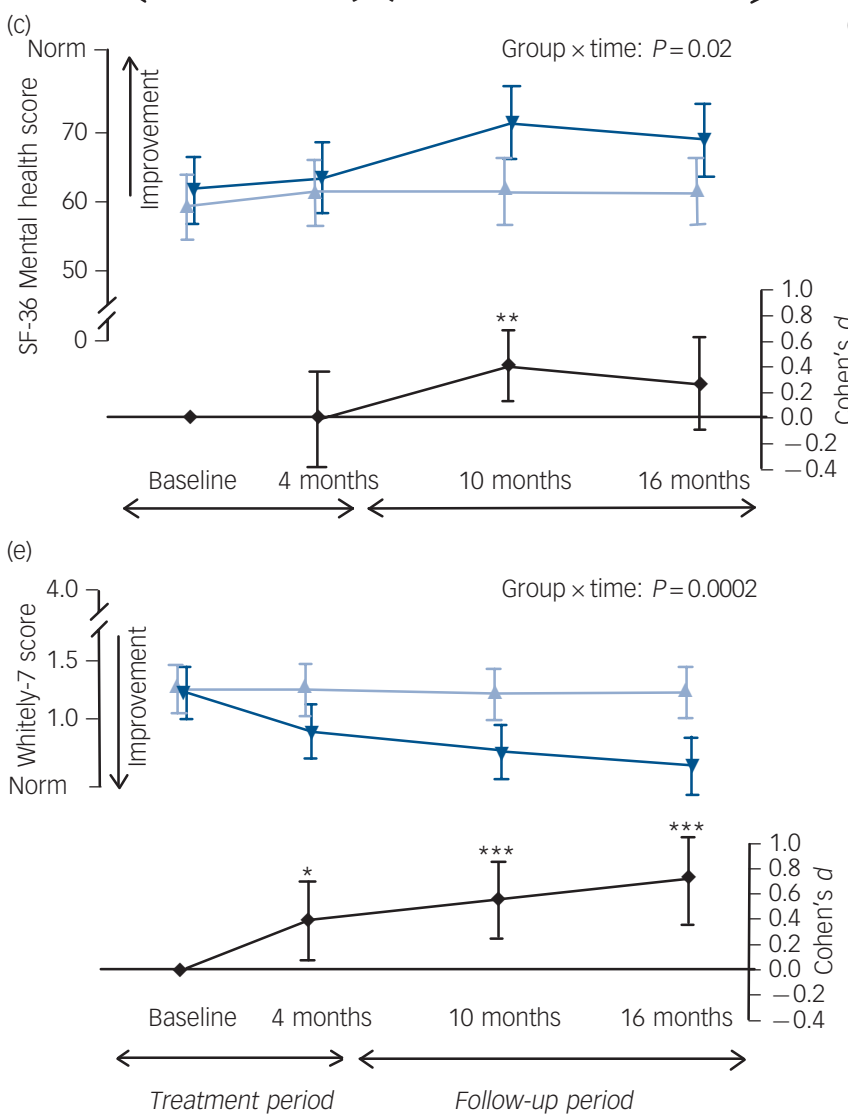

(f)

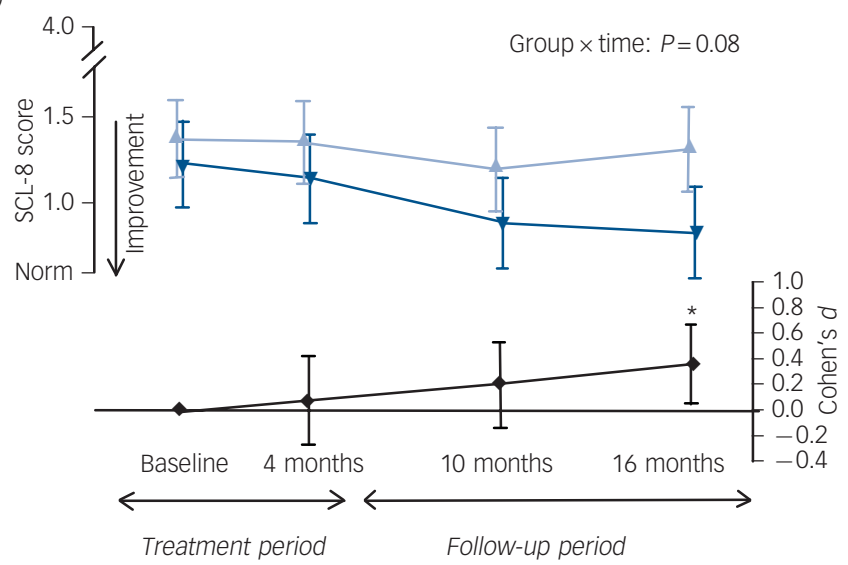

Fig. 3 Effect of the intervention on (a) physical health and (b-f) secondary outcomes.

The top two curves of each graph give the mean values and $95 \%$ confidence intervals for the intervention and usual care groups; the $P$-value is for the overall group $\times$ time interaction (adjusted mixed model, Wald $\chi^{2}$ test); this test indicates whether the illness course differs between groups. Comparison effect sizes (adjusted Cohen's $d$ ) are shown as the lower curve, calculated as adjusted between-group difference in mean change since baseline, divided by pooled standard deviation at baseline. Positive effect sizes favour the intervention. Error bars indicate 95\% confidence intervals; asterisks indicate level of significance for $d \neq 0$. (a) Physical health (primary outcome): aggregate score of three Short Form Health Survey (SF-36) subscales (physical function, bodily pain and vitality). (b) Social functioning. (c) Mental health. Higher scores in these three graphs indicate better health; norm indicates mean of the general Danish population. (d) Physical symptoms scored with the 90-item Symptom Checklist - Revised. (e) IIIness worry measured with the 7-item Whiteley scale. (f) Anxiety/depression, measured with the 8-item Symptom Checklist. The three latter graphs show illness severity scores, with lower scores indicating less severe illness (for illness worry, norm indicates mean of patients with well-defined medical conditions). ${ }^{*} P<0.05,{ }^{*} P<0.01,{ }^{* * *} P<0.001$.

baseline to 16 months on the SF-36 PCS was 6.2 points (95\% CI 2.5-9.9, $P=0.001)$. Participants allocated to STreSS improved by 5.6 points $(95 \%$ CI $2.5-8.7, P<0.001)$, whereas participants allocated to usual care remained substantially unchanged $(-0.6$ points, $95 \%$ CI -2.7 to $1.4 ; P=0.54)$. Comparison effect sizes for the SF-36 PCS were 0.37 (95\% CI 0.18-0.58), 0.43 (95\% CI $0.24-0.62)$ and 0.61 (95\% CI $0.26-0.98)$ at 4 months, 10 months and 16 months respectively (data not shown in Fig. 3).

\section{Secondary outcomes}

Figure 4 gives the proportions of patients in each treatment group who had improved by at least 4 points (treatment response) or
8 points (large improvement) on the primary outcome from baseline to 16 months. The relative risk for treatment response was 1.8 (95\% CI 1.1-3.2, $P=0.03$ ) in favour of STreSS. The NNT to achieve one additional treatment response with STreSS compared with enhanced usual care was 5 ( $95 \%$ CI $2-38, P=0.03$ ). Over half $(56 \%)$ of patients in the usual care group reported their physical health to be worse than before randomisation, which was the case for only a quarter $(25 \%)$ of the STreSS group.

The difference between the treatment groups increased over time, with statistical significance for all secondary outcomes (see Fig. 3) except for the anxiety and depression severity score. Although patients allocated to usual care did not improve at 


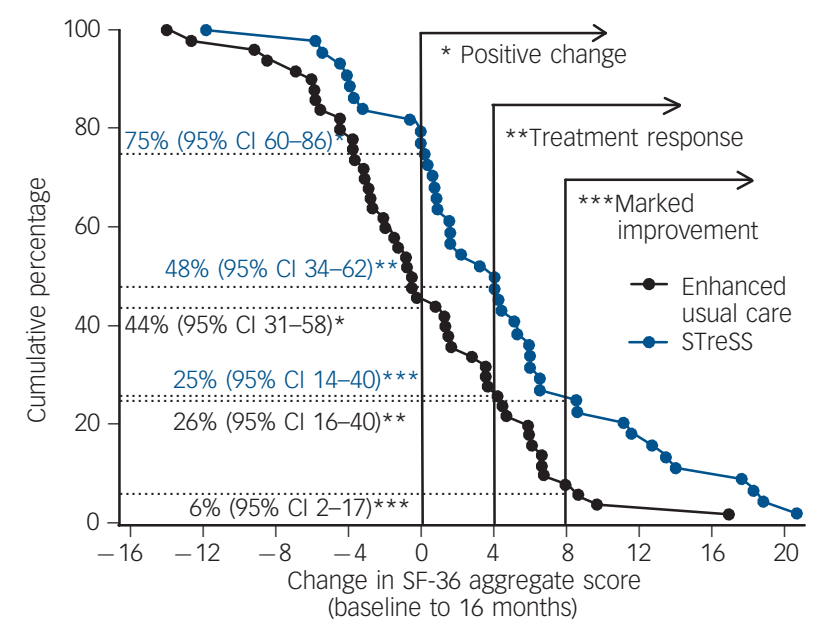

Fig. 4 Improvement in physical health from baseline to 16 months in both groups.

Plots present the observed data, with each dot representing the observed change score for an individual patient who provided data at 16 months. Numbers indicate the percentage of patients $(95 \% \mathrm{Cl})$ in each group whose self-reported physical health had improved at a given level from baseline to 16 months. Vertical lines and asterisks indicate these levels of improvement: * positive change, i.e. change scores $\geqslant 0$ points, $* *$ treatment response, i.e. change scores $\geqslant 4$ points or 0.5 s.d. unit; ***marked improvement, i.e. change scores $\geqslant 8$ points or 1.0 s.d. unit.

any time point on any of the secondary outcomes, patients allocated to STreSS experienced an immediate reduction in physical symptoms and illness worry, which was sustained or even increased from 4 months to 16 months. However, differences in social functioning and mental health between the two groups were first observable at 10 months. This sequence suggests that improvement in social functioning and mental health might be a consequence of symptom relief, rather than a mechanism of change.

\section{Discussion}

For patients with severe functional somatic syndromes combined under the unifying category of multi-organ bodily distress syndrome, the STreSS intervention produced a greater improvement in self-reported physical health than that achieved by usual care enhanced by a thorough initial clinical assessment. The treatment effect was of clinical significance and was sustained at follow-up. We also noted evidence that the intervention led to greater improvements than usual care in patients' social functioning and mental health, and to marked reductions in physical symptoms and illness worry.

To the best of our knowledge, this is the first report of a single CBT approach applicable to the management of a group of patients with different severe functional somatic syndromes conducted in secondary care. Most of the trials reported to date have included patients with a single functional somatic syndrome diagnosis, ${ }^{8,12-16,34}$ or comprising a special group such as Gulf War veterans. ${ }^{35}$ On the other hand, studies of somatoform disorders in mental health settings are often conducted in highly selected patient populations and rarely include participants with formal diagnoses of functional somatic syndromes. ${ }^{36}$ Our study suggests that patients with the unifying diagnosis of bodily distress syndrome can feasibly be treated together regardless of the functional somatic syndrome diagnosis they have been previously given.
The current evidence for CBT is largely limited to short-term follow-up, and recent meta-analyses have called for measurement of longer-term outcomes in trials of treatment for functional somatic syndromes. ${ }^{12,13,36}$ Our study provides that evidence; the positive effect induced by the STreSS intervention was sustained during the 12-month follow-up period. Moreover, since STreSS is a group treatment, the psychiatrists delivering it spent fewer hours per patient than required for individually delivered treatments. ${ }^{34}$ The group format was well accepted by the majority of patients, and the rates of withdrawal and effect sizes observed in this trial are comparable to those reported in previous studies. ${ }^{8,12-16,34,36}$ The STreSS intervention may therefore be more cost-efficient than individually delivered CBT, although this needs further investigation.

Compared with psychological interventions delivered in mental health settings, an important strength of the STreSS intervention is its integrated approach. ${ }^{7,8}$ This treatment provides patients with a positive and evidence-based understanding of their illness that aims to transcend the mind-body dualism inherent in the current diagnostic classifications, ${ }^{37,38}$ and that contrasts with the usual approach to management, which tends to suggest either physical or mental disease. Although the behavioural and cognitive treatment elements of STreSS are targeted at maintaining psychosocial and lifestyle factors that can be influenced by behavioural interventions, ${ }^{39}$ psychoeducation about functional somatic syndromes specifically acknowledges known biological factors in their aetiology. ${ }^{40-42}$ Patients learn that although their somatic symptoms are best understood as pathophysiological responses to prolonged or severe mental or physical stress on the basis of genetic susceptibility, both psychological and behavioural factors are known to be involved in the initiation and maintenance of those symptoms, ${ }^{39,43}$ and that these factors therefore are the target of the treatment. This balanced conceptualisation may not only increase patients' motivation to engage in psychological treatment, but also help them to accept their vulnerability to developing new somatic symptoms, and to adapt their lifestyle accordingly in order to prevent relapse.

\section{Limitations}

The findings of this trial have to be discussed against the background of its potential limitations: we included only patients with multi-organ bodily distress syndrome, and hence with multiple functional somatic symptoms. Multiple symptoms are a marker of illness severity, ${ }^{1,18}$ and therefore the efficacy of STreSS for patients with less severe functional somatic syndromes remains unclear. Furthermore, although we included consecutively referred patients from both primary and secondary care, the study sample is not fully representative of people with severe functional somatic syndromes, since only young and middle-aged patients were included. Therefore, we do not know whether STreSS would be effective in older people. A final point regarding generalisability is the fact that all patients in the study underwent a thorough clinical assessment prior to treatment allocation. We therefore cannot be sure that STreSS would be as effective in people who are not offered this kind of assessment, which might have increased the participants' motivation to engage in the intervention.

The efficacy of STreSS in this trial may have been influenced by the fact that treatment was carried out by a few highly skilled psychiatrists, and our findings therefore require replication. However, the fact that patients in the comparison group also received a specific intervention (the clinical assessment) is likely to have improved their outcome, and potentially diminished the treatment effect observed. The interpretation of the effect sizes reported in this study is limited by the fact that the primary 
outcome was an aggregate measure of three SF-36 scales that has not yet been used in other clinical trials. However, we found similar effect sizes on the standard SF-36 PCS. Finally, although we were able to demonstrate a clear treatment effect, with the point estimate reaching the pre-specified clinically important difference of 4 points, the wide confidence intervals do not definitely establish a clinically significant treatment effect. A much larger sample would be needed to determine this.

The STreSS intervention is composed of several components, but the design of the study does not allow us to determine whether one component is more active than another in achieving change. Accordingly, our aim was simply to assess whether the whole complex intervention as delivered was acceptable and effective in improving functioning and health-related quality of life. However, it is unlikely that the observed effect was driven by systematic differences in the usual care delivered in both treatment groups, since we found no such difference in use of primary care and mental health services, or in use of prescription pain medication and antidepressants during the 16-month study period.

We are not yet able to provide cost comparison data, and the cost-effectiveness of the STreSS intervention therefore remains currently unclear. A thorough cost-effectiveness analysis that also includes societal costs such as sick leave and social benefits is in progress.

\section{Implications}

This study suggests that it is feasible and acceptable to patients and physicians to implement a single cognitive-behavioural group treatment for people with different severe functional somatic syndromes, thereby offering a pragmatic and effective treatment approach for a large group of people who are usually regarded as difficult to treat and often described as unwilling to accept psychological treatment. This novel unified treatment approach may be preferable to the implementation of different specialised treatments for single functional somatic syndromes in each secondary medical care service.

\section{Further research}

This trial is only a first step to evaluating an effective management strategy for patients with severe functional somatic syndromes. The STreSS intervention needs to be compared with other active treatments, and the possible additional effect of pharmacotherapy also requires investigation. Two further trials will address these questions. The results of our study need to be confirmed in large, multicentre trials designed to explore the effectiveness and costeffectiveness of STreSS or similar complex interventions in people with functional somatic syndromes before widespread implementation is justified. These trials should be powered to allow subgroup analyses of the effect of STreSS on different types of functional somatic syndromes and on patients with and without psychiatric comorbidity.

In summary, STreSS provides a promising unified approach to the management of people with severe functional somatic syndromes that overcomes existing shortcomings in organisation of care.

\section{Funding}

The study was funded by Central Denmark Region, the Aarhus University Hospital Research Initiative, the A.P. Møller Foundation for the Advancement of Medical Science and the Medical Association for the County of Aarhus.

\section{Acknowledgements}

We thank Morten Frydenberg for thoughtful advice regarding study design; Lone Fjordback for her help with patient care; Jane Walker for helpful comments on the manuscript; our colleagues and the staff from the Research Clinic for Functional Disorders for their invaluable participation; and the patients for their participation in this study.

\section{Andreas Schröder, MD, PhD, Emma Rehfeld, MD, Eva ørnbøl, MSC, Research Clinic for Functional Disorders and Psychosomatics, Aarhus University Hospital, Aarhus, Denmark; Michael Sharpe, MD, Psychological Medicine Research, Aarhus, Denmark; Michael Sharpe, MD, Psychological Medicine Research,
Department of Psychiatry, University of Oxford, UK; Rasmus W. Licht, MD, PhD, Mood Disorders Research Unit, Aarhus University Hospital, Risskov; Per Fink, MD, PhD, DrMedSc, Research Clinic for Functional Disorders and Psychosomatics, Aarhus University Hospital, Aarhus, Denmark \\ Correspondence: Dr Andreas Schröder, Research Clinic for Functional Disorders and Psychosomatics, Aarhus University Hospital, DK-8000 Aarhus C, Denmark. E-mail: andreas.schroeder@aarhus.rm.dk \\ First received 13 Jul 2011, final revision 12 Dec 2011, accepted 19 Dec 2011}

\section{Appendix}

\section{Specialised Treatment for Severe Bodily Distress Syndromes (STresS)}

Module 1: Introduction (week 1)

Enhancing motivation to deal with painful and disabling bodily symptoms. Full acknowledgement of patients' suffering. Introduction to cognitivebehavioural therapy. Introduction of group members.

Module 2: Bodily symptoms and their interpretation (week 2) Registering and differentiating bodily symptoms. Challenging inflexible symptom attributions.

Module 3: Illness perceptions, stress response and treatment goals (week 3)

Diagnostic labels for and subtypes of bodily distress syndromes. Biological, psychological and social factors contributing to the development and maintenance of bodily distress. Impact of negative illness perceptions. Defining individual treatment goals for each patient.

Module 4: Negative automatic thoughts and dysfunctional behaviours (week 4)

(Re-)connecting bodily symptoms with emotions, thoughts and behaviours. For each patient, identification of perpetuating factors (thoughts and behaviours) that contribute to disability.

Module 5: Cognitive distortions and emotional awareness (week 6) Ongoing work with the connection of bodily symptoms, emotions, thoughts and behaviours. Identification of cognitive distortions. Construction of alternative responses. Enhancing emotional awareness.

Module 6: From illness behaviour to health behaviour (I) (week 8) Ongoing work with the connection of bodily symptoms, emotions, thoughts and behaviours. Looking back: connecting life events and bodily distress. Looking forward: boosting pleasurable activities.

Module 7: From illness behaviour to health behaviour (II) (week 10) Restoring sleep, balanced diet and physical exercise. Evaluating social network and interpersonal relationships. Evaluating work status. Revision and adjustment of individual treatment goals.

Module 8: Becoming your own therapist; relapse prevention (week 12)

Adapting lifestyle to improved functioning. Recapitulation of dysfunctional thoughts and behaviours, and construction of alternative beliefs. Providing problem-solving skills. Drawing up individual treatment manual for possible relapse. 
Module 9: How to maintain learned skills and coping strategies (week 16)

Review of concepts taught and skills learned in the STresS programme. Definition of individual goals for the next 3 months. Recapitulation and farewell.

\section{Treatment delivery}

Each module consists of $3.5 \mathrm{~h}$ of therapy. Treatment is delivered by two psychiatrists to groups of seven to nine patients. Each patient is allocated a contact psychiatrist who is primarily responsible for his or her treatment. An English version of the treatment manual is available from the authors upon request (and will be available at www.functionaldisorders.dk).

\section{References}

1 Simon G, Gater R, Kisely S, Piccinelli M. Somatic symptoms of distress: an international primary care study. Psychosom Med 1996; 58: 481-8.

2 Wessely S, Nimnuan C, Sharpe M. Functional somatic syndromes: one or many? Lancet 1999; 354: 936-9.

3 Whitehead LC. Quest, chaos and restitution: living with chronic fatigue syndrome/myalgic encephalomyelitis. Soc Sci Med 2005; 62: 2236-45.

4 Barsky AJ, Orav EJ, Bates DW. Somatization increases medical utilization and costs independent of psychiatric and medical comorbidity. Arch Gen Psychiatry 2005; 62: 903-10.

5 Bass C, Peveler R, House A. Somatoform disorders: severe psychiatric illnesses neglected by psychiatrists. Br J Psychiatry 2001; 179: 11-4.

6 Creed F. Should general psychiatry ignore somatization and hypochondriasis? World Psychiatry 2006; 5: 146-50.

7 Sharpe M, Carson A. 'Unexplained' somatic symptoms, functional syndromes, and somatization: do we need a paradigm shift? Ann Intern Med 2001; 134: 926-30.

8 Henningsen P, Zipfel S, Herzog W. Management of functional somatic syndromes. Lancet 2007; 369: 946-55.

9 Fink P. Surgery and medical treatment in persistent somatizing patients. J Psychosom Res 1992; 36: 439-47.

10 Glombiewski JA, Rief W, Bosner S, Keller H, Martin A, Donner-Banzhoff N. The course of nonspecific chest pain in primary care: symptom persistence and health care usage. Arch Intern Med 2010; 170: 251-5.

11 Fink $P$. The use of hospitalizations by persistent somatizing patients. Psycho Med 1992; 22: 173-80.

12 Zijdenbos IL, de Wit NJ, van der Heijden GJ, Rubin G, Quartero AO. Psychological treatments for the management of irritable bowel syndrome. Cochrane Database Syst Rev 2009; CD006442.

13 Price JR, Mitchell E, Tidy E, Hunot V. Cognitive behaviour therapy for chronic fatigue syndrome in adults. Cochrane Database Syst Rev 2008; CD001027.

14 Kroenke K. Efficacy of treatment for somatoform disorders: a review of randomized controlled trials. Psychosom Med 2007; 69: 881-8.

15 Glombiewski JA, Sawyer AT, Gutermann J, Koenig K, Rief W, Hofmann SG. Psychological treatments for fibromyalgia: a meta-analysis. Pain 2010; 151 280-95

16 White PD, Goldsmith KA, Johnson AL, Potts L, Walwyn R, DeCesare JC, et al Comparison of adaptive pacing therapy, cognitive behaviour therapy, graded exercise therapy, and specialist medical care for chronic fatigue syndrome (PACE): a randomised trial. Lancet 2011; 377: 823-36.

17 Shir $Y$, Fitzcharles MA. Should rheumatologists retain ownership of fibromyalgia? J Rheumatol 2009; 36: 667-70.

18 Fink $\mathrm{P}$, Toft $\mathrm{T}$, Hansen MS, Ornbol E, Olesen F. Symptoms and syndromes of bodily distress: an exploratory study of 978 internal medical, neurological, and primary care patients. Psychosom Med 2007; 69: 30-9.

19 Fink $P$, Schröder A. One single diagnosis, bodily distress syndrome succeeded to capture ten diagnostic categories of functional somatic syndromes and somatoform disorders. J Psychosom Res 2010; 68: 415-26.
20 Perera R, Heneghan C, Yudkin P. Graphical method for depicting randomised trials of complex interventions. BMJ 2007; 334: 127-9.

21 World Health Organization. SCAN: Schedules for Clinical Assessment in Neuropsychiatry, Version 2.1. WHO Division of Mental Health, 1998.

22 Nielsen JM, Vedsted P, Olesen F. The postgraduate psychiatric training of general practitioners: a questionnaire survey in Aarhus County. Ugeskr Laeger 2002; 164: 895-9.

23 Ware J, Kosinski M, Gandek B. SF-36 Health Survey: Manual and Interpretation Guide. Quality Metric, 2005.

24 Mease $\mathrm{P}$, Arnold LM, Bennett R, Boonen A, Buskila D, Carville $\mathrm{S}$, et al. Fibromyalgia syndrome. J Rheumatol 2007; 34: 1415-25.

25 Ware JE, Kosinski M. SF-36 Physical and Mental Health Summary Scales: A Manual for Users of Version 1, Second Edition. Quality Metric, 2001.

26 Norman GR, Sloan JA, wyrwich KW. Interpretation of changes in healthrelated quality of life: the remarkable universality of half a standard deviation. Med Care 2003; 41: 582-92.

27 Ware JE, Bayliss MS, Rogers WH, Kosinski M, Tarlov AR. Differences in 4-year health outcomes for elderly and poor, chronically ill patients treated in HMO and fee-for-service systems. Results from the Medical Outcomes Study. JAMA 1996; 276: 1039-47.

28 Fink $\mathrm{P}$, Ewald $\mathrm{H}$, Jensen J, Sørensen $\mathrm{L}$, Engberg $\mathrm{M}$, Holm $\mathrm{M}$, et al. Screening for somatization and hypochondriasis in primary care and neurological in-patients: a seven-item scale for hypochondriasis and somatization. J Psychosom Res 1999; 46: 261-73.

29 Derogatis LR, Cleary PA. Confirmation of the dimensional structure of the SCL-90: A study in construct validation. J Clin Psychol 1977; 33: 981-9.

30 Fink $P$, Ørnbøl E, Huyse FJ, De Jonge $P$, Lobo A, Herzog T, et al. A brief diagnostic screening instrument for mental disturbances in general medica wards - the SCL-8 Scale. A European multi-centre study. J Psychosom Res 2004; 57: 17-24.

31 Schröder A, Ørnbøl E, Licht RW, Sharpe M, Fink P. Outcome measurement in functional somatic syndromes: SF-36 summary scores and some scales were not valid. J Clin Epidemiol 2012; .65: 30-41.

32 Hann M, Reeves D. The SF-36 scales are not accurately summarised by independent physical and mental component scores. Oual Life Res 2008; 17 $413-23$

33 Walwyn $\mathrm{R}$, Roberts $\mathrm{C}$. Therapist variation within randomised trials of psychotherapy: implications for precision, internal and external validity. Stat Methods Med Res 2010; 19: 291-315.

34 Allen LA, Woolfolk RL, Escobar Jl, Gara MA, Hamer RM. Cognitive-behavioral therapy for somatization disorder: a randomized controlled trial. Arch Intern Med 2006; 166: 1512-8.

35 Donta ST, Clauw DJ, Engel CC, Guarino P, Peduzzi P, Williams DA, et al. Cognitive behavioral therapy and aerobic exercise for Gulf War veterans' illnesses: a randomized controlled trial. JAMA 2003; 289: 1396-404.

36 Kleinstauber M, Witthoft M, Hiller W. Efficacy of short-term psychotherapy for multiple medically unexplained physical symptoms: a meta-analysis. Clin Psychol Rev 2011; 31: 146-60.

37 Schröder A, Fink P. The proposed diagnosis of somatic symptom disorders in DSM-V: two steps forward and one step backward? J Psychosom Res 2010; 68: 95-6.

38 Feinstein AR. The Blame-X syndrome: problems and lessons in nosology, spectrum, and etiology. J Clin Epidemiol 2001; 54: 433-9.

39 Deary $\mathrm{V}$, Chalder $\mathrm{T}$, Sharpe $\mathrm{M}$. The cognitive behavioural model of medically unexplained symptoms: a theoretical and empirical review. Clin Psychol Rev 2007; 27: 781-97.

40 Bradley LA. Pathophysiologic mechanisms of fibromyalgia and its related disorders. J Clin Psych 2008; 69 (suppl 2): 6-13.

41 Clauw DJ. Potential mechanisms in chemical intolerance and related conditions. Ann NY Acad Sci 2001; 933: 235-53.

42 Wood PB. Neuroimaging in functional somatic syndromes. Int Rev Neurobiol 2005; 67: 119-63.

43 Rief W, Broadbent E. Explaining medically unexplained symptoms - models and mechanisms. Clin Psychol Rev 2007; 27: 821-41. 